Original Paper

\title{
HistopathologicAl ASSESSMENT OF ORAL LEUKOPLAKiA. OSTEONECTIN AS POSSIBLE BIOMARKER FOR FURTHER DIAGNOSTICS
}

\author{
Irena Duś-Ilnicka ${ }^{1}$, Malgorzata Radwan-Oczko ${ }^{1}$, Hanna Gerber ${ }^{2}$, Agnieszka Halon Han $^{3}$
}

${ }^{1}$ Oral Pathology Department, Faculty of Dentistry, Wroclaw Medical University, Wroclaw, Poland

${ }^{2}$ Department and Clinic of Maxillofacial Surgery, Faculty of Dentistry, Wroclaw Medical University, Wroclaw, Poland ${ }^{3}$ Department of Pathomorphology and Oncological Cytology, Faculty of Dentistry, Wroclaw Medical University, Wroclaw, Poland

\begin{abstract}
Clinical evaluation of oral leukoplakia (OL), confirmed by the histological evaluation of the suspected area, provides the gold standard for diagnostics of this pathology. The aim of present study was to encrypt the significance of the histopathological results (oral intraepithelial neoplasia - OIN, WHO 2005, Ljubljana classification systems) of OL. The usefulness of osteonectin as a biomarker of changes in the oral cavity epithelium was evaluated.

IRS Score to evaluate osteonectin (SPARC - secreted protein acidic and rich in cysteine) production in oral mucous tissues was modified, with the aim of adapting the diagnostic measurements to the OL cell environment. In total, 37 formalin-fixed, paraffin-embedded (FFPE) blocks from patients with clinically diagnosed OL, and 29 FFPE blocks from patients with OSCC were evaluated. The OIN and system from Ljubljana were compared, to adjudicate which was most compatible with WHO 2005 histopathological assessment.

Increased production of SPARC was observed, with the progression in severity of pathological changes in the oral mucosa, from simple hyperplasia, through dysplasia, to OSCC. The WHO 2005 and the OIN classification systems can be applied interchangeably.
\end{abstract}

Key words: osteonectin, SPARC, oral leukoplakia, oral neoplasms.

\section{Introduction}

Oral leukoplakia (OL) is classified by the WHO as an oral potentially malignant disorder (OPMD), with the possibility of transformation to oral squamous cell carcinoma (OSCC) in $0.13 \%$ to $34 \%$ of cases, depending on the source of the data given $[1,2]$. Oral leukoplakia, with an average worldwide prevalence of $2 \%$, is a multifactorial disorder. Among the possible risk factors for its development and progression, smoking habit and alcohol abuse, or Candida albicans suprainfection, are most commonly mentioned. All those might trigger the genetic alterations leading to OSCC $[3,4,5,6]$. Relatively little is known about OSCC as a disease affecting patients worldwide, and therefore the disease is still an open chapter of the recent research $[6,7]$. Hence, proper diagnostics of OPMD, which leads to the development of OSCC, is of great relevance. 
Alcohol consumption might have mutagenic and carcinogenic properties, because of toxic ethanol metabolite production - acetaldehyde - and its action on the oral mucous membrane. A lot of research was conducted to find the main cause for the development of these pathologies, but there is still a little evidence of molecular changes leading to disease progression. This condition varies from one geographical area to another, although it was found that the higher risk of neoplastic transformation begins in anatomical areas of the oral cavity covered with non-keratinised mucosa $[8,9,10,11]$.

Proper diagnosis of OPMD relies on the patient's medical history, clinical assessment, and histopathological diagnosis of oral epithelium changes [12]. According to the World Health Organisation (WHO), histopathological evaluation remains the gold standard for OL diagnostics [13]. After histopathological diagnostics of the cell progression, the clinical treatment schedule might be chosen, when the potential presence, or grade of dysplasia is considered [12], but the evaluation systems used by pathologists differ between laboratories. Changes in the field of results presented by pathologists influence the possibility of proper clinical evaluation of the disease, because of non-coherent names of grades between classification systems. Proper understanding of classification systems grades, are crucial for the clinical proceedings after the histopathological evaluation. In this article the conformity of two classification systems the OIN system and the Ljubljana system - were compared with the WHO 2005 system [14] (Table I). All listed classification systems are used interchangeably, and the use of a specific system depends on the pathomorphologists' preferences or regional requirements for histopathological reporting. The WHO classification system is considered as a gold standard for histological evaluation and is updated frequently. The latest update, WHO 2017, provided the extension of mild dysplasia grade to the grade previously called "squamous hyperplasia" in the WHO 2005 classification $[14,15]$. Because such a change might be of great importance for proper treatment of patients, it shows the need for unification of the classification systems of oral changes, and for the search for modern biomarkers reflecting the actual state of the patient's disease.

Secreted protein acidic and rich in cysteine (SPARC), also known as osteonectin and BM-40 (basement membrane protein 40) is a phosphorylated glycoprotein with a molecular weight from 32 to $43 \mathrm{kDa} \llbracket 16$, $17,18]$. The presence of this protein has been demonstrated in tissues that undergo frequent renewal, such as chondral tissue or the epithelium of the gastrointestinal tract. In addition, this protein appears in endothelial cells in response to damage, as well as in regions of active bone remodeling [18], and is observed in patients with periodontitis during periodontal

Table I. Comparison between WHO systems from 2005, Ljubljana System, and OIN system

\begin{tabular}{lcc}
\hline ORAL INTRAEPITHELIAL NEOPLASIa (OIN) & WHO, 2005 & LubLANA (SIL) \\
\hline n/a & Squamous cell hyperplasia & Simple squamous cell hyperplasia \\
\hline OIN 1 & Mild dysplasia & Basal/parabasal cell hyperplasia \\
\hline OIN 2 & Moderate dysplasia & Atypical hyperplasia \\
\hline OIN 3 & Severe dysplasia & Atypical hyperplasia \\
\hline & Carcinoma in situ & Carcinoma in situ \\
\hline
\end{tabular}

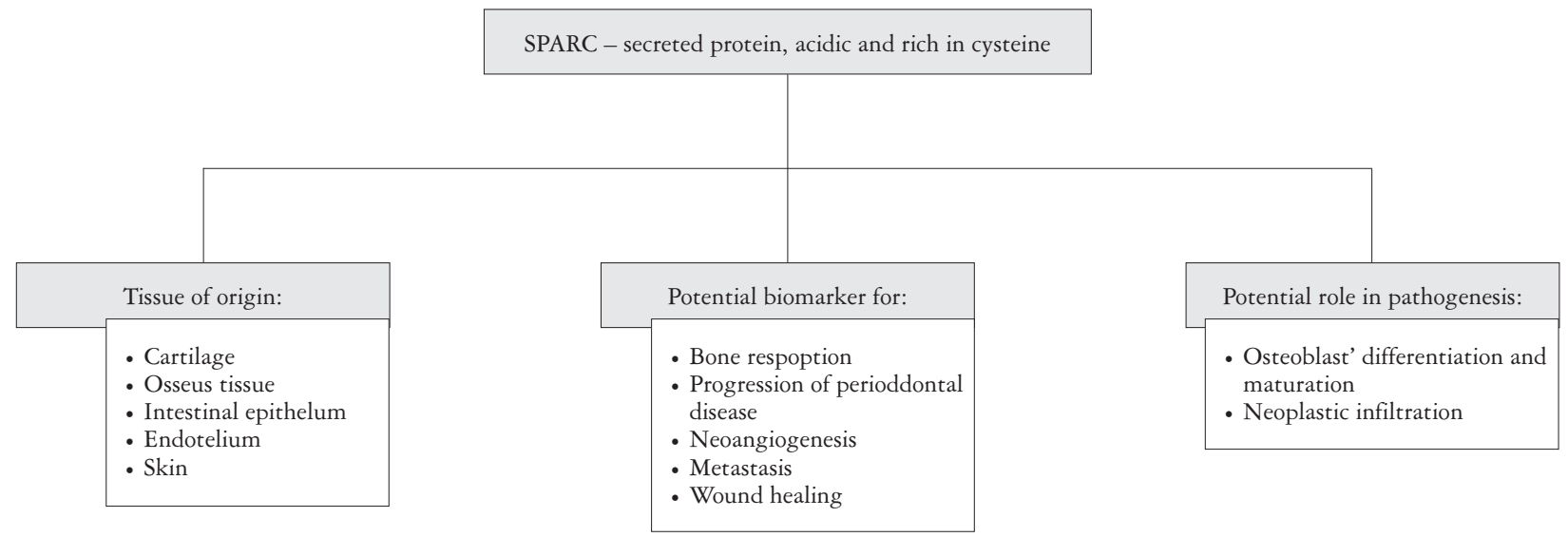

Fig. 1. Characteristics of SPARC protein 
repair processes [19]. Figure 1 presents a summary of SPARC's characteristics. It is suggested that osteonectin may participate in cancer progression. Protein activity causes increased metalloproteinase-2 (MMP-2) activity in human tumour tissues, contributing to the development of neoangiogenesis [18]. The specific function of SPARC in neoplastic processes in OSCC has not yet been thoroughly described, and it is most important that it be discovered, because in other types of cancer cells its function may have a stimulatory or inhibitory effect on the malignancy process [17]. It has been suggested that in the processes of malignant oral epithelium transformation, the processes of increased tissue remodelling may be involved, intensified under the influence of various repair processes stimuli. This leads to the conclusion that a possible role of SPARC is as an indicative progression marker in oral pathologies. When we search for such keywords as "SPARC AND oral leukoplakia" or "osteonectin AND oral leukoplakia" in the medical search engine - PubMed.gov - there is only one publication available in the results. Therefore, the basis for the use of this molecular marker seems open to discussion. In this study, the expression of osteonectin in the precancerous state of OL (along with the division into particular histopathological stages of oral mucous biopsies) was evaluated in 31 cases, and in 29 cases of OSCC.

\section{Material and methods}

\section{Tissue specimens and patients' medical history}

Formalin-fixed, paraffin-embedded (FFPE) blocks with biopsies from the oral mucous membrane were obtained from the archives of the Department of Pathomorphology of a local hospital. Formalin-fixed paraffin-embedded blocks prepared from biopsies taken from patients of the Oral Pathology Department, with clinical suspicion of OL, were afterwards processed for histopathology. The leading pathologist re-diagnosed FFPE samples with the use of three different classification systems - Oral Intraepithelial Neoplasia (OIN), the classification system from Ljubljana, and the WHO 2005.

Patients' clinical charts from the Department and Clinic of Maxillofacial Surgery of the local hospital were analysed with respect to all compliance acts and the anonymity of all individuals. The approval of the local Ethics Committee was obtained for the research.

\section{Immunohistochemical staining}

Tissue material consisted of archived oral mucosa biopsies fixed in $10 \%$ buffered formalin and paraffin-embedded:
- 37 blocks from 31 different patients from diverse areas of OL,

- 29 blocks from 29 patients with oral neoplasms.

From the prepared FFPE blocks, the samples of $5 \mu \mathrm{m}$ thickness were obtained, with the use of $\mathrm{mi}$ crotome apparatus. Afterwards, deparaffinization in xylene was carried out, and material was put on SuperFrost slides (Super Frost Plus Menzel GLASSER, Braunschweig, Germany). For immunohistochemistry reaction SPARC Mouse Monoclonal Antibody was used (AON-5031, No.sc-73472, Santa Cruz Biotechnology, US). The described process was carried out with the use of Target Retrival Solution and PT Link Rinse Station at pH 9 (temp. $97^{\circ} \mathrm{C}, 20 \mathrm{~min}$ ). The samples were washed in TBS and incubated with original antibody in an automated IHC Dako Link 48 Autostainer system (room temperature, $20 \mathrm{~min}$ ). VVisualisation was proceeded in EnVison FLEX (DakoCytomation) reagent, and samples were dyed in haematoxylin, according to the guidelines of the manufacturers. The primary staining was accompanied by a negative control.

\section{Evaluation of SPARC protein expression}

Production of SPARC protein by immunohistochemical reaction was evaluated in various stages of oral mucous membrane pathologies - from OL, in comparison with SPARC production in OSCC. The negative control was the non-invaded margin of the oral epithelium of the oral mucous membrane. Expression of SPARC protein was evaluated in Yamada's semi-quantitative scale of immunoreactivity (Immunoreactive Score - IRS) [17] in the author's modification, based on Remmele's immunoreactivity scale [20]. In the evaluation of SPARC expression, two parameters were considered together: the percentage of cells showing positive cytoplasmic reaction and the intensity of colour reaction.

Definitive rating of immunohistochemical reaction was included in a five-grade semi quantitative modified IRS scale, for the purpose of this publication referred to as the IRS Score. The described parameters of immunohistochemical evaluation are presented in Table II.

Table II. ImmunoreactiveYamada Score, IRS in the author's modification

\begin{tabular}{lcc}
\hline $\begin{array}{l}\text { Percentage of Cells with } \\
\text { COlOur Reaction }\end{array}$ & Reaction & Score \\
\hline $\begin{array}{l}\text { No cells, or number of cells } \\
\text { under }<5 \%\end{array}$ & Negative & 0 \\
\hline $5-25 \%$ positive cell reaction & Weak & 1 \\
\hline $26-50 \%$ positive cell reaction & Moderate & 2 \\
\hline $51-80 \%$ positive cell reaction & Strong & 3 \\
\hline$>80 \%$ positive cell reaction & Very strong & 4 \\
\hline
\end{tabular}


Table III. Characteristics of patients

\begin{tabular}{lcc}
\hline & OSCC & LEUKOPLAKIA \\
\hline Number of patients & 29 & 31 \\
\hline Sex & & $61 \% / 39 \%$ \\
\hline \multicolumn{1}{l}{ Men/Women } & $59 \% / 41 \%$ & 57.5 \\
\hline Average age & 65.1 & $26 \%$ \\
\hline Smoking habit: & $41 \%$ & $6 \%$ \\
\hline Patients who gave up smoking during the treatment & $17 \%$ & $16 \%$ \\
\hline Patients smoking more than 10 cigaretts per day & $21 \%$ & $13 \%$ \\
\hline Previous oncological treatment in oral cavity area & $17 \%$ & $6 \%$ \\
\hline Alcohol habit - patient's declaration & $24 \%$ & $10 \%$ \\
\hline General health problems & $14 \%$ & $32 \%$ \\
\hline Diabetis & $34 \%$ & 0 \\
\hline Hipertension & $24 \%$ & $6.5 \%$ \\
\hline Varicose veins of the lower limbs & $21 \%$ & \\
\hline Gastroenterological disorders & & \\
\hline
\end{tabular}

Table IV. Localisation of leukoplakia and squamous cell carcioma changes in oral mucosa

\begin{tabular}{|c|c|c|c|c|c|c|c|c|c|}
\hline & & \multicolumn{7}{|c|}{ LOCALISATION } & \multirow[t]{2}{*}{ Total } \\
\hline & & Tongue & GINGIVA & LIP & С HeEK & $\begin{array}{c}\text { ANGLE } \\
\text { OF THE MOUTH }\end{array}$ & $\begin{array}{l}\text { HARD } \\
\text { PALATE }\end{array}$ & $\begin{array}{l}\text { THE BOTTOM } \\
\text { OF THE MOUTH }\end{array}$ & \\
\hline Number & OscC & 10 & 9 & 0 & 4 & 0 & 0 & 2 & 25 \\
\hline$\%$ & & $40.00 \%$ & $36.00 \%$ & $0.00 \%$ & $16.00 \%$ & $0.00 \%$ & $0.00 \%$ & $8.00 \%$ & \\
\hline Number & $\begin{array}{l}\text { Leuko- } \\
\text { plakia }\end{array}$ & 4 & 6 & 3 & 10 & 1 & 3 & 2 & 29 \\
\hline$\%$ & & $13.79 \%$ & $20.69 \%$ & $10.34 \%$ & $34.48 \%$ & $3.45 \%$ & $10.34 \%$ & $6.90 \%$ & \\
\hline
\end{tabular}

\section{Results}

\section{Patients' characteristics}

A statistically significantly higher average age of patients with OSCC was found in comparison to patients with $\mathrm{OL}(\mathrm{p}=0.0150)$. Patients general characteristics and medical history results are presented in Table III. Referring to the location of the lesion, a slightly more frequent occurrence of OL has been demonstrated on the buccal mucosa and for OSCC on the tongue of patients. The results of the total percentage of localizations in the oral cavity of both pathologies are shown in Table IV.

\section{Histopathological evaluation}

\section{Oral neoplasms group}

In the group of 29 oral neoplasms, according to the WHO classification, the following were identified:
- 13 biopsies of Carcinoma planoepithelialekeratodes,

- 12 biopsies of Carcinoma planoepithelialeakeratodes,

- 4 biopsies of Carcinoma verrucosum.

Among the C. planoepithelialekeratodes and akeratodes, the grade of histopathological malignancy (Grading, G) was evaluated as follows:

- $8 \mathrm{Gl}$ cancers in the highest degree of differentiation (the lowest grade of malignancy),

- 14 G2 cancers in the middle degree of differentiation (the moderate grade of malignancy),

- 4 G3 cancers with the lowest degree of differentiation (the highest degree of malignancy).

\section{Oral lenkoplakia group}

This section compares the results of the comparative histopathological assessment of oral mucous biopsies with the use of two different histological systems Oral Intraepithelial Neoplasia (OIN) (Table VI) and the system of grading from Ljubljana (Table VII) in reference to the WHO 2005 system.

The Wilcoxon statistical test was used in the study. Comparison of the results obtained by 
Table V. Evaluation of the results for SPARC expression in oral leukoplakia and oral squamous cell carcinoma

\begin{tabular}{lcccccc}
\hline & GROUP & SPARC 0 & SPARC1 & SPARC 2 & SPARC 3 & SPARC 4 \\
\hline Numeric value & OSCC & 3 & 9 & 8 & 4 & 2 \\
\hline$\%$ & & $11.54 \%$ & $34.62 \%$ & $30.77 \%$ & $15.38 \%$ & $7.69 \%$ \\
\hline Numeric value & Leukoplakia & 20 & 9 & 1 & 0 & 0 \\
\hline$\%$ & & $66.67 \%$ & $30.00 \%$ & $3.33 \%$ & $0.00 \%$ & $0.00 \%$ \\
\hline
\end{tabular}

Table VI. Comparison of histopathological results obtained after applying the WHO classification in comparison with the OIN system

\begin{tabular}{cccccc}
\hline \multicolumn{7}{c}{ OIN } \\
\hline \multirow{3}{*}{ WHO } & & 0 & 1 & 2 & 3 \\
\cline { 2 - 6 } & 0 & 17 & 2 & 0 & 0 \\
\cline { 2 - 6 } & 1 & 0 & 4 & 0 & 0 \\
\cline { 2 - 6 } & 2 & 0 & 0 & 3 & 0 \\
\hline
\end{tabular}

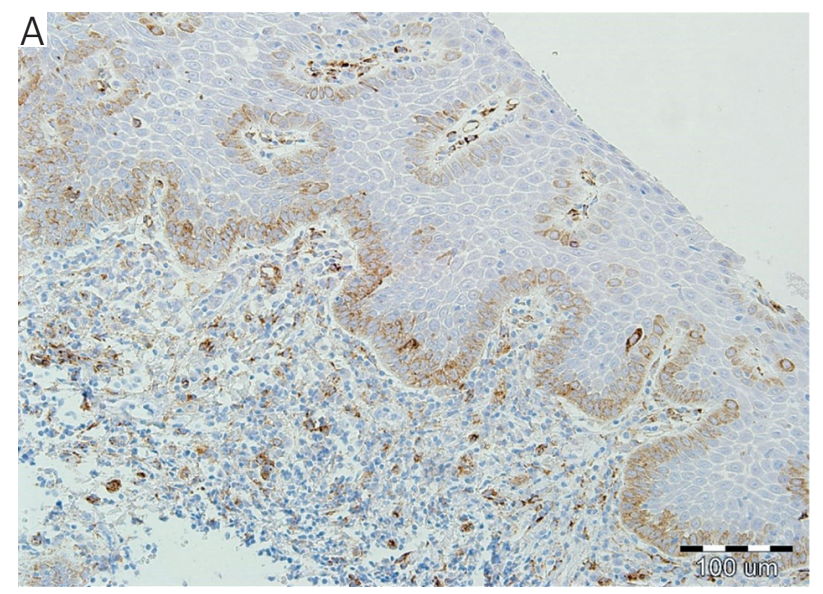

Figure 2. Image A) represents simple hyperplasia of the oral mucosa epithelium. IHC reaction with SPARC antibody Score 1. Image B) represents squamous cell carcinoma G1. IHC reaction with SPARC antibody - Score 3

histopathological analysis of the material showed that the OIN system was the most consistent with the WHO classification system, with a coefficient of $\mathrm{p}=0.180$.

\section{SPARC immunohistochemical staining}

Expression of SPARC protein with the use of immunohistochemistry was evaluated in the case of 26 patients with diagnosed oral neoplasms. Lack of expression (SCORE 0) was evaluated in $11.54 \%$ of patients, SCORE 1 expression was evaluated in $34.63 \%$, SCORE 2 expression was evaluated in $30.77 \%$, SCORE 3 expression was evaluated in $15.38 \%$, and the $4^{\text {th }}$ grade of expression - SCORE 4 - was evaluated in $7.69 \%$ of all patients.

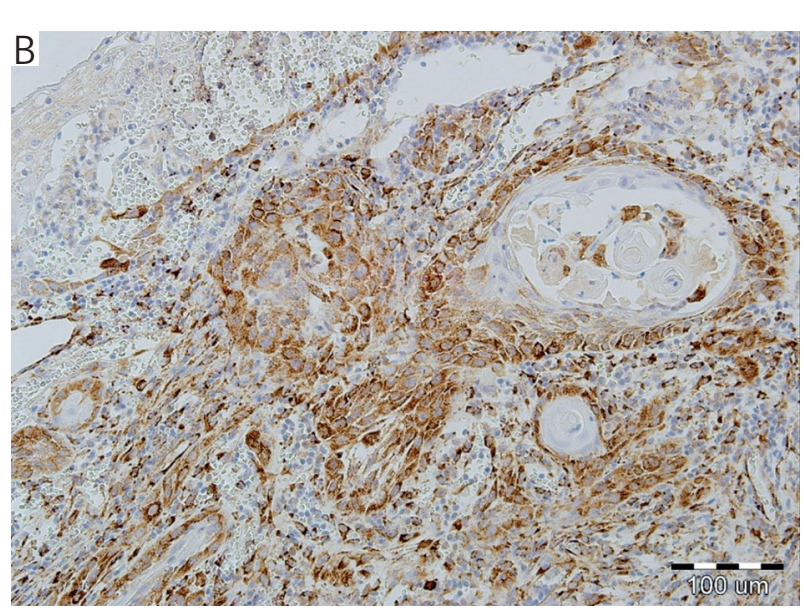

Table VII. Comparison of histopathological results obtained after applying the WHO classification compared to the system from Ljubljana

\begin{tabular}{lccccc}
\hline \multicolumn{7}{c}{ LjUbljana } \\
\hline \multirow{3}{*}{ WHO } & & 0 & 1 & 2 & 3 \\
\cline { 2 - 6 } & 0 & 15 & 2 & 0 & 0 \\
\cline { 2 - 6 } & 1 & 0 & 2 & 2 & 0 \\
\cline { 2 - 6 } & 2 & 0 & 0 & 3 & 0 \\
\hline
\end{tabular}

Expression of SPARC protein with the use of immunohistochemistry was evaluated in 30 patients with clinically diagnosed leukoplakia. Lack of expression (SCORE 0) was evaluated in $66.67 \%$, SCORE 1 (Fig. 2) expression was evaluated in $30.00 \%$, SCORE 2 expression was evaluated in 1 case, and SCORE 3 (Fig. 2) and 4 were not present in the whole group of OL. All the described results are shown in Table V.

The Spearman test was used for statistical evaluation of all obtained results. With the progression of severity of the oral mucosa pathological changes (from mucosal hyperplasia to C. planoepitheliale), increased production of osteonectin was observed. Thus, a correlation was found in the production 


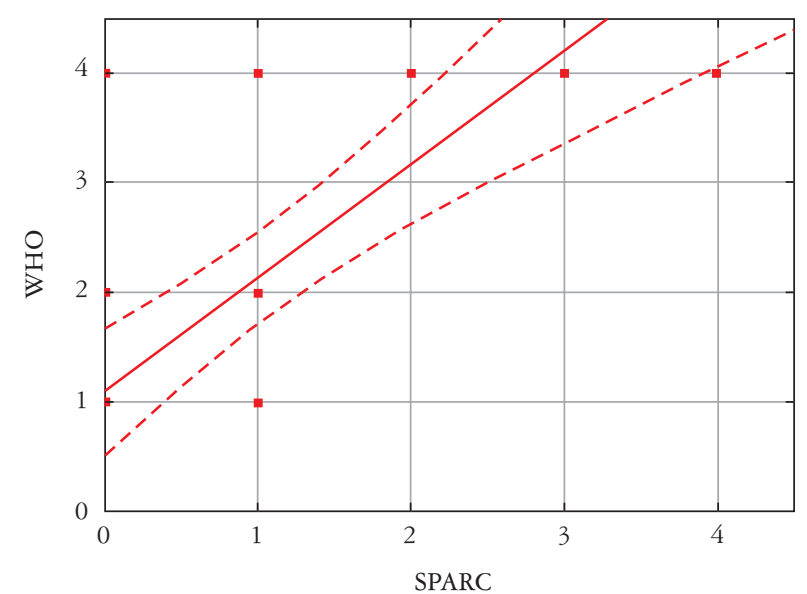

Fig. 3. Correlation between SPARC protein production and stages of oral mucous progression

of SPARC protein among oral mucous pathologies. This correlation is shown in Fig. 3.

\section{Discussion}

Leukoplakia is the most common oral, potentially malignant, disorder, with prevalence of $4.11 \%$ based of the meta-analysis of Mello et al. [12] Taking into consideration the patients' gender, in the present study men were more often affected by OL than women, and represented $61 \%$ of all patients with clinically diagnosed OL. According to Shearston et al., this premalignant lesion more often affects smokers, and usually shows its first clinical symptoms in the $4^{\text {th }}$ decade of life [2]. The average age of patients with OL from the Lower Silesia region was 57.5 years, and that places the results of this research in the first standard deviation of the results obtained by Ang et al. [6] and in line with those of Shearston et al. [2]. In total $26 \%$ of patients from the OL group of the presented research declared having a smoking habit. Common sites of OL include the tongue and buccal mucosa [2], and in the present study the most common site of OL was buccal mucosa.

OSCC is ranked as the eighth most common cancer in males worldwide, but in females it is not on the list of top ten cancers [7]. From all patients affected with OSCC in the present research, men represented 59\%. According to the Polish National Cancer Registry, malignant neoplasms of the tongue are rare and constitute about $0.5 \%$ incidence of all reported malignant neoplasm cases among men in Poland, and about $0.2 \%$ of all reported malignant neoplasms cases among women [21]. In the present research, this localisation of OSCC was most frequent in all patients, reaching $40 \%$ of all analysed OSCC cases. The highest incidence of tongue cancer occurs in patients during the sixth to seventh decade of life, according to the Polish National Cancer Registry [21].
In our research the average age of all patients with OSCC was 65.1 years.

Histopathological reporting of OL is subjective and external quality assurance is crucial here [22]. This study provided evaluation of two different classification systems - OIN and Ljubljana - both used by pathologists during histopathological evaluation of oral biopsies. The aim was to compare both classification systems with the WHO 2005 system. Ljubljana classification is considered to be an alternative OPMD assessment, but it was developed for use in laryngeal pathologies [22]. For that reason, the term "dysplasia" in not present, and the term "atypical hyperplasia" is used instead [22]. This change has to be taken into consideration when the results are received, because of the significance of dysplasia diagnostics in further clinical proceedings. In the present article the conclusion was reached that the highest coherence with the WHO 2005 system was received by the use of OIN classification. Oral intraepithelial neoplasia assessment is based on the Squamous Intraepithelial Neoplasia/dysplasia (SIN/dysplasia) classification from 2005, first created for the uterine cervix diagnostics, and afterwards extended to other mucosae [14, 23]. The OIN system grades lesions as high and low grade, and concordance with the WHO 2005 system presented in this paper might show the reasoning for the cut-off point for dysplasia in oral mucous membrane samples [14].

Given the latest available data, regarding the possibility of malignant transformation, OL might be a pathological condition of medium transformation. Shearston et al. showed that this incidence in the Australian population was $1.49 \%$, with an average time of malignant transformation of 5.2 years [2]. The term "oral leukoplakia" might be used to cover clinical changes in the oral cavity, but confirmation in the histopathological results is needed, and therefore collaboration between a pathologist and a dentist is crucial [24]. Proper diagnostics should lead to histopathological assessment of changed oral mucosa to distinguish the grade and presence of dysplasia by evaluation of FFPE [24]. As many researchers show, traditional histopathological diagnostics of OL might not be enough to identify all lesions that possibly might be undergoing malignant transformation process. Therefore, there is a need of new histopathological and molecular biomarkers, that could be used to detect this process with greater specificity [2]. The function of osteonectin as a key regulator is to control the cell proliferation, migration, and survival. Despite growing interest in the role of SPARC in various types of cancer, information on protein involvement in the issue of tumor formation and progression is contradictory [16]. Studies by many authors prove the relationship of SPARC protein with the malignancy of breast cancer, melanoma, osteosar- 
coma, glioblastomas and bladder cancer. On the other hand, the inhibitory effect of the glycoprotein on the development of ovarian cancer has been demonstrated, as well as the use of SPARC in the prognosis of pancreatic adenocarcinoma or colon cancer $[16,17$, 18]. Osteonectin as a predictive marker in dentistry is still a matter of research. Animal models are used to broaden the understanding of the role of SPARC in periodontitis [19] and its association with cancerisation of oral squamous epithelium, as discussed in [17], along with research performed in order to evaluate the correlation between SPARC expression in tumour cells in OSCC with the worst pattern of invasion [25]. Demonstration of whether the aforementioned dependence could be used in the progression of leukoplakia-type neoplastic states was one of the aims of this study.

It was confirmed that SPARC may be an indicator of mutations during the initial stages of epithelial carcinogenesis, however, the usefulness of this biomarker ends at this stage, and it was not indicated as a predictive factor in cancer prognosis $[17]$. The described research of Yamada et al. led the authors of the present study to diagnose the expression of SPARC protein in primary stages of oral cell pathology progression in order, to evaluate its possible role as a predictive marker.

During the process of the research presented herein, a higher expression of SPARC was demonstrated in the form of pathological changes in the oral mucosa, which progressed from different histopathological cell stages in biopsies from OL to OSCC, compared to the healthy tissue margins. Based on this study it may be concluded that osteonectin production is induced by dysplastic cells in the initial stages of neoplastic development, it stopped at this stage and was not an indicator of malignancy of oral neoplasms. In order to clearly determine this issue, further studies should be carried out on larger groups of patients.

Acknowledgment of grant support: this research was performed with grant support from Wroclaw Medical University, No. Pbmn112.

The authors declare no conflicts of interest.

\section{References}

1. Hassona Y, Scully C, Almangush A, et al. Oral potentially malignant disorders among dental patients: a pilot study in Jordan. Asian Pac J Cancer Prev 2014; 15: 10427-10431.

2. Shearston K, Fateh B, Tai S, et al. Malignant Transformation Rate of Oral Leukoplakia in an Australian population. J Oral Pathol Med 2019; jop.12899.

3. de Menezes RF, Bergmann A, Thuler LCS. Alcohol Consumption and Risk of Cancer: a Systematic Literature Review. Asian Pacific J Cancer Prev 2014; 14: 4965-4972.

4. Silveira M, Dedivitis R, Queija D, Nascimento P. Quality of Life in Swallowing Disorders after Nonsurgical Treatment for Head and Neck Cancer. Int Arch Otorhinolaryngol 2014; 19: 046-054.

5. Boffetta P, Ye W, Adami HO, et al. Risk of cancers of the lung, head and neck in patients hospitalized for alcoholism in Sweden. Br J Cancer 2001; 85: 678-682.

6. Ang XM, Au PCK, Kwok K, et al. Quality of Life in Patients with Oral Leukoplakia. J Oral Pathol Med 2019; jop.12897.

7. Malik UU, Zarina S, Pennington SR. Oral squamous cell carcinoma: Key clinical questions, biomarker discovery, and the role of proteomics. Arch Oral Biol 2016; 63: 53-65.

8. Brouns ER, Baart JA, Bloemena E, et al. The relevance of uniform reporting in oral leukoplakia: Definition, certainty factor and staging based on experience with 275 patients. Med Oral Patol Oral Cir Bucal 2013; 18: e19-e26.

9. Bouquot JE, Speight PM, Farthing PM. Epithelial dysplasia of the oral mucosa - diagnostic problems and prognostic features. Curr Diagnostic Pathol 2006; 12: 11-21.

10. Jaber MA, Porter SR, Speight P, et al. Oral epithelial dysplasia: clinical characteristics of western European residents. Oral Oncol 2003; 39: 589-596.

11. Martorell-Calatayud A, Botella-Estrada R, Bagán-Sebastián JV, et al. Oral Leukoplakia: Clinical, Histopathologic, and Molecular Features and Therapeutic Approach. Actas DermoSifiliográficas (English Ed) 2009; 100: 669-684.

12. Mello FW, Miguel AFP, Dutra KL, et al. Prevalence of oral potentially malignant disorders: A systematic review and meta-analysis. J Oral Pathol Med 2018; 47: 633-640.

13. El-Naggar AK, Chan JKC, Rubin Grandis J, et al. International Agency for Research on Cancer. WHO Classification of Head and Neck Tumours. https://publications.iarc.fr/BookAnd-Report-Series/Who-Iarc-Classification-Of-Tumours/ Who-Classification-Of-Head-And-Neck-Tumours-2017. Accessed July 3, 2019.

14. Ranganathan K, Kavitha L. Oral epithelial dysplasia: Classifications and clinical relevance in risk assessment of oral potentially malignant disorders. J Oral Maxillofac Pathol 2019; 23: 19-27.

15. Barnes L, Eveson JW, Reichart P (eds.). Pathology and Genetics of Head and Neck Tumours. Available at: http:// publications.iarc.fr/Book-And-Report-Series/Who-Iarc-Classification-Of-Tumours/Pathology-And-Genetics-Of-HeadAnd-Neck-Tumours-2005.

16. Aquino G, Sabatino R, Cantile M, et al. Expression Analysis of SPARC/Osteonectin in Oral Squamous Cell Carcinoma Patients: From Saliva to Surgical Specimen. Biomed Res Int 2013; 2013: 1-9

17. Yamada T, Ohno S, Kitamura N, et al. SPARC is associated with carcinogenesis of oral squamous epithelium and consistent with cell competition. Med Mol Morphol 2015; 48: 129-137.

18. Futyma K, Kubiatowski T, Rózyńska K, et al. Decreased osteonectin and fibronectin gene expression in endometrial cancer cancer as a prognostic marker. Ginekol Pol 2009; 80: 907-913.

19 Trombetta JM, Bradshaw AD. SPARC/osteonectin functions to maintain homeostasis of the collagenous extracellular matrix in the periodontal ligament. J Histochem Cytochem 2010; 58: 871-879.

20. Remmele W, Stegner HE. [Recommendation for uniform definition of an immunoreactive score (IRS) for immunohistochemical estrogen receptor detection (ER-ICA) in breast cancer tissue]. Pathologe 1987; 8: 138-140.

21. Wojciechowska U, Didkowska J. Zachorowania i zgony na nowotwory złośliwe w Polsce. Krajowy Rejestr Nowotworów Centrum Onkologii - Instytut Im. Marii Skłodowskiej-Curie. Dostępne na stronie: Http://Onkologia.Org.Pl/Raporty/ Dostęp z Dnia 03/07/2019. http://onkologia.org.pl/raporty/ [Accessed July 3, 2019]. 
22. Warnakulasuriya S, Reibel J, Bouquot J, Dabelsteen E. Oral epithelial dysplasia classification systems: Predictive value, utility, weaknesses and scope for improvement. J Oral Pathol Med 2008; 37: 127-133.

23. Küffer R, Lombardi T. Premalignant lesions of the oral mucosa. A discussion about the place of oral intraepithelial neoplasia (OIN). Oral Oncol 2002; 38: 125-130.

24. van der Waal I. Oral leukoplakia, the ongoing discussion on definition and terminology. Med Oral Patol Oral Cir Bucal 2015; 20: e685-e692.

25. Jing Y, Jin Y, Wang Y, et al. SPARC promotes the proliferation and metastasis of oral squamous cell carcinoma by PI3K/ AKT/PDGFB/PDGFR $\beta$ axis. J Cell Physiol 2019; 10.1002/ jcp. 28205

\section{Address for correspondence}

Irena Duś-Ilnicka

Oral Pathology Department

Faculty of Medicine

Wroclaw Medical University

Krakowska 26

50-425 Wroclaw, Poland

e-mail: irena.dus@gmail.com, irena.dus-ilnicka@umed.wroc.pl 